\title{
Comorbid mental disorders among clients in addiction treatment: The costs of care
}

\author{
Karen Urbanoski ${ }^{1,2}$, Jürgen Rehm ${ }^{1,2,3,4}$, Shannon Lange ${ }^{1}$, and Svetlana Popova ${ }^{1,2,3,5}$ \\ ${ }^{1}$ Social and Epidemiological Research Department, Centre for Addiction and Mental Health (CAMH), Toronto, ON, Canada \\ ${ }^{2}$ Dalla Lana School of Public Health, University of Toronto, Toronto, ON, Canada \\ ${ }^{3}$ Institute of Medical Science, University of Toronto, Toronto, ON, Canada \\ ${ }^{4}$ Epidemiological Research Unit, Klinische Psychologie and Psychotherapie, Technische Universität Dresden, Dresden, Germany \\ ${ }^{5}$ Factor-Inwentash Faculty of Social Work, University of Toronto, Toronto, ON, Canada
}

\begin{abstract}
Aims: To compare the volume and costs of services for clients with and without comorbid mental disorders in an addiction treatment system.

Design: Secondary data analysis.

Setting: Administrative data for the fiscal year 2010-2011 were abstracted from the Drug and Alcohol Treatment Information System (DATIS).

Participants: Data represents all clients entering publicly funded services in Ontario in the fiscal year 2010-2011 $(N=41,953)$.

Measures: Service costs were calculated using recent estimates of the cost per outpatient visit and day of residential care. Average numbers of visits/days per client, with and without mental disorders, were compared across gender and age subgroups.

Findings: Overall, 22\% of clients treated in 2010-2011 reported a past-year mental disorder. The costs of services for these clients were estimated to be between $\$ 18.5$ and $\$ 40.3$ million (in Canadian dollars), or $20 \%$ of the total service costs for the year. The largest difference in average volume of care was seen for residential treatment, where having a mental disorder was associated with seven fewer days of care. On average, during the period studied, among those without mental disorders, men spent 20 more days in residential care than did women.
\end{abstract}

Conclusions: At the system-level in Ontario, clients with mental disorders did not account for a disproportionate amount of the total costs of care during the year. While these findings may signal a mismatch between the needs of clients with co-occurring disorders and their use of services, it is also possible that such clients receive additional care in other treatment sectors. Further study is warranted to explore potential unmet needs among clients with co-occurring disorders in addiction treatment.

Substance abuse is a major public health problem. In 2002, an estimated $11 \%$ of Canadians reported past-year substance use problems or dependence (Rush et al., 2008). Taking into account costs associated with health care, law enforcement, and loss of productivity in the workplace as a result of premature death and disability, the overall social cost of substance abuse was estimated to be $\$ 39.8$ billion $^{1}$ in Canada in 2002 (Popova et al., 2006; 2007; Rehm et al., 2006; 2007).

It is clear from community-based and clinical research that a considerable proportion of people with substance use

\footnotetext{
${ }^{1}$ All cost figures presented in the present study are in Canadian dollars.
}

disorders experience co-occurring mental disorders (Grant et al., 2004; Kessler et al., 1997; Rush et al., 2008; Teesson et al., 2000). Particularly in clinical contexts, evidence suggests that the majority of clients have a history of both types of disorders (Adamson, Todd, Sellman, Huriwai, \& Porter, 2006; Castel, Rush, Urbanoski, \& Toneatto, 2006; Chan, Dennis, \& Funk, 2008; Langås, Malt, \& Opjordsmoen, 2012). In addition to presenting with a more complex array of problems, clients with co-occurring disorders are at risk of poorer substance-related and psychological outcomes following treatment (Baker et al., 2007; Bradizza, Stasiewica, \& Paas, 2006; Compton, Cottler, Jacovs, Ben-Abadallah, \& Spitznagel, 2003; Weisner, Matzger, \& Kaskutas, 2003; Wusthoff, Waal, Ruud, \& Gråwe, 2011). 
Despite the clear policy and clinical implications, there is a dearth of research examining the implications of cooccurring disorders for the course and costs of addiction treatment. Findings are equivocal on the link between cooccurring mental disorders and participation or retention in addiction treatment (Carroll, Power, Bryant, \& Rounsaville, 1993; Chi, Satre, \& Weiser, 2006; Pani et al., 2011; Stark, 1992; Verthein, Degkwitz, Haasen, \& Krausz, 2005). One U.S. study found elevated costs of outpatient and inpatient addiction treatment among clients with co-occurring mental disorders (Hoff \& Rosenheck, 1999). Given the reliance on factors such as treatment length and costs on system-level and local administrative and organizational issues, there is a need for research examining the implications of cooccurring disorders in different jurisdictions and sectors of care.

The present study examines differences in the volume and costs of services between clients with and without mental disorders in specialized addiction treatment in Ontario, Canada.

\section{Method}

\section{Data source}

Administrative data were obtained from the Drug and Alcohol Treatment Information System (DATIS), which monitors the number and characteristics of clients receiving specialized treatment for alcohol and other drug problems across Ontario (estimated population is 13.4 million). All publicly funded services are mandated by the provincial government to routinely provide data to DATIS. This includes approximately 200 programs administered by 170 agencies (Ogborne, Braun, \& Rush, 1998; Rotondi \& Rush, 2012). Some agencies provide a specific type of service (e.g., withdrawal management, outpatient counseling), while others provide a comprehensive mix of services. Opiate substitution therapy is provided through physician offices and health clinics, which do not report to DATIS. Therefore, methadone maintenance treatment clients are not represented in DATIS unless they also receive counseling at a participating agency. Services are delivered free of charge to Ontario residents, covered by the province's universal health insurance plan. Client demographic characteristics, information on substance use, and other treatment-related factors are collected at the beginning of the treatment episode, while data on service use are entered at discharge. Data are stored on a central server located at the Centre for Addiction and Mental Health in Toronto, Ontario, Canada. Agency-level participation has been over 95\% since 2000 .

\section{Study population}

All episodes of treatment spanning the fiscal year 2010 2011 were extracted for analysis $(N=41,953$ clients with 91,333 admissions). For the purposes of generating accurate estimates of the volume and costs for different service types (i.e., outpatient, residential, and withdrawal management), each admission corresponds to a specific type of care; that is, a transition from residential to an outpatient service was counted as two episodes of care. Also, multiple admissions for a given client within each type of service were included. Approximately 15\% of outpatient episodes ( $N=5,681$ ) were excluded because they did not have any visits associated with them, and therefore did not contribute any information to the service volume or cost estimates.

\section{Data elements}

All data were collected as part of routine clinical assessment. Clients were asked whether they had been diagnosed with a mental disorder by a qualified mental health professional in the past year. Age at admission was generated from date of birth. Sex was recorded as male or female (a small number of clients reported their gender as "other" and were excluded from the present analysis). Service type (outpatient, residential, and residential withdrawal management) was generated automatically in DATIS based on a unique program identifier. Program staff entered the number of outpatient visits, days in residential treatment, and days in residential withdrawal management at discharge. For episodes of care that began prior to or ended after the fiscal year 2010-2011, only those visits and days that occurred within the study period were counted.

\section{Analysis}

We estimated the volume and costs of services by past-year mental disorder status, separately by service type and for the system overall. Upper and lower estimates of costs for units of care were obtained from an ongoing provincial study. The cost for specialized outpatient treatment ranged from $\$ 60$ to $\$ 109$ per visit and for residential treatment (including residential withdrawal management) ranged from \$138 to \$314 per resident day in Canada in 20102011 (Martin et al., July 12, 2012, personal communication). These unit costs were estimated based on the costs reported by five Local Health Integration Networks across Ontario. The overall estimates are inclusive of the cost of supervision, facility costs, salaries, and other sundry expenses. The ranges reported account for the differences in costs incurred due to the number of spots/beds available (capacity), whether the treatment is hospital-based or community-based, the intensity of activities provided, and staff professionalism (which affects both their salaries and the scope of the staff complement). These cost estimates were applied to the number of visits or days of care and summed across service types to provide estimates of the total costs for those with and without mental disorders. A small number of clients $(N=2,324$, $5.5 \%$ ) provided discrepant information on past-year diagnoses of mental disorders across admissions during the study period; that is, they reported both having and not having been diagnosed with a mental disorder in separate admissions made during 2010-2011. Services received following admissions in which mental disorders were reported are included in the cost estimates for those with mental disorders, and vice versa. Note that the counts of clients within each service type are overestimates as a result of this overlap. 
To assess whether a past-year mental disorder appeared to be associated with a disproportionate level of service use or costs, we compared the proportion of total costs accounted for by clients with mental disorders to the system-level prevalence of mental disorders (i.e., as a proportion of clients and admissions). Finally, to identify potential differences across subgroups of the treatment population, we compared the average volume of services (visits or days) per client with and without mental disorders across sex and broad age groups (24 or younger, 25 to 39, 40 to 54, and 55 and over).

\section{Results}

The total system-level costs for specialized addiction treatment in 2010-2011 were estimated to be between $\$ 90.4$ and $\$ 197.4$ million (Table 1). Overall, 22\% of clients treated during this year reported past-year mental disorders. These clients accounted for $18 \%$ of admissions and $20 \%$ of the service costs during the year-an estimated
$\$ 18.5$ to $\$ 40.3$ million. Figure 1 depicts the costs by mental disorder status and service type.

\section{Outpatient services}

The total cost of outpatient services for clients with mental disorders was estimated to be between $\$ 4.0$ and $\$ 7.3$ million (Table 1). Clients with mental disorders accounted for $21 \%$ of the caseload in outpatient services, regardless of whether multiple admissions per client were included, and $22 \%$ of the volume or costs of services delivered during 2010-2011.

The average number of outpatient visits per client in 20102011 was 11.9 and 10.7 among those with and without past-year mental disorders, respectively. Among men, the average number of visits per client was 11.0 for those with and 10.3 for those without mental disorders. For women, these figures were slightly higher, at 12.9 and 11.4 visits per client, respectively. Figure 2 shows the distribution of average visits per client by age. The average number of visits per client was slightly lower for clients aged 24 or younger, particularly for those without mental disorders.

\section{Table 1}

Overview of the volume and costs of addiction treatment, by past-year mental disorders in Ontario, Canada, fiscal year 20102011

\begin{tabular}{|c|c|c|c|c|}
\hline & Outpatient & Residential & Withdrawal & Overall \\
\hline \multicolumn{5}{|l|}{ Clients With Mental Disorders } \\
\hline Number of Clients & 5,635 & 1,766 & 3,409 & $9,343^{\mathrm{a}}$ \\
\hline Number of Admissions & 6,523 & 2,119 & 8,242 & 16,884 \\
\hline Number of Visits & 67,023 & $\mathrm{n} / \mathrm{a}$ & $\mathrm{n} / \mathrm{a}$ & 67,023 \\
\hline Number of Days & $\mathrm{n} / \mathrm{a}$ & 84,916 & 20,296 & 105,212 \\
\hline Cost (lower estimate) & $\$ 4,021,380$ & $\$ 11,718,408$ & $\$ 2,800,803$ & $\$ 18,540,591$ \\
\hline Cost (upper estimate) & $\$ 7,305,507$ & $\$ 26,663,624$ & $\$ 6,372,841$ & $\$ 40,341,972$ \\
\hline \multicolumn{5}{|c|}{ Clients Without Mental Disorders } \\
\hline Number of Clients & 21,666 & 5,964 & 13,707 & $34,934^{\mathrm{a}}$ \\
\hline Number of Admissions & 24,790 & 7,203 & 42,456 & 74,449 \\
\hline Number of Visits & 231,095 & $\mathrm{n} / \mathrm{a}$ & $\mathrm{n} / \mathrm{a}$ & 231,095 \\
\hline Cost (lower estimate) & $\$ 13,865,700$ & $\$ 45,115,788$ & $\$ 12,828,727$ & $\$ 71,810,215$ \\
\hline Cost (upper estimate) & $\$ 25,189,355$ & $\$ 102,654,764$ & $\$ 29,190,002$ & $\$ 157,034,121$ \\
\hline Total Number of Clients & 27,301 & 7,730 & 17,116 & $41,953^{\mathrm{a}, \mathrm{b}}$ \\
\hline Total Number of Admissions & 31,313 & 9,322 & 50,698 & 91,333 \\
\hline Total Number of Visits & 298,118 & $\mathbf{n} / \mathbf{a}$ & $\mathbf{n} / \mathbf{a}$ & 298,118 \\
\hline Total Number of Days & $\mathbf{n} / \mathbf{a}$ & 411,842 & 113,257 & 525,099 \\
\hline Total Cost (lower estimate) & $\$ 17,887,080$ & $\$ 56,834,196$ & $\$ 15,629,530$ & $\$ 90,350,806$ \\
\hline Total Cost (upper estimate) & $\$ 32,494,862$ & $\$ 129,318,388$ & $\$ 35,562,844$ & $\$ 197,376,094$ \\
\hline
\end{tabular}

n/a: Not applicable

${ }^{\mathrm{a}}$ The total number of clients does not equal the sum of clients across treatment modalities, as some clients received care in multiple modalities during the year

${ }^{\mathrm{b}} 2,324$ (5.5\%) of clients provided different answers to the question about mental disorder diagnoses across admissions within the year; within service categories, they are represented in the client counts for both subgroups (with and without mental disorders) 
Figure 1

Costs (lower and upper estimates) associated with specialized addiction treatment among clients with and without lifetime mental disorder in Ontario, Canada in 2010/2011

$$
\square \text { mental disorder } \quad \square \text { no mental disorder }
$$

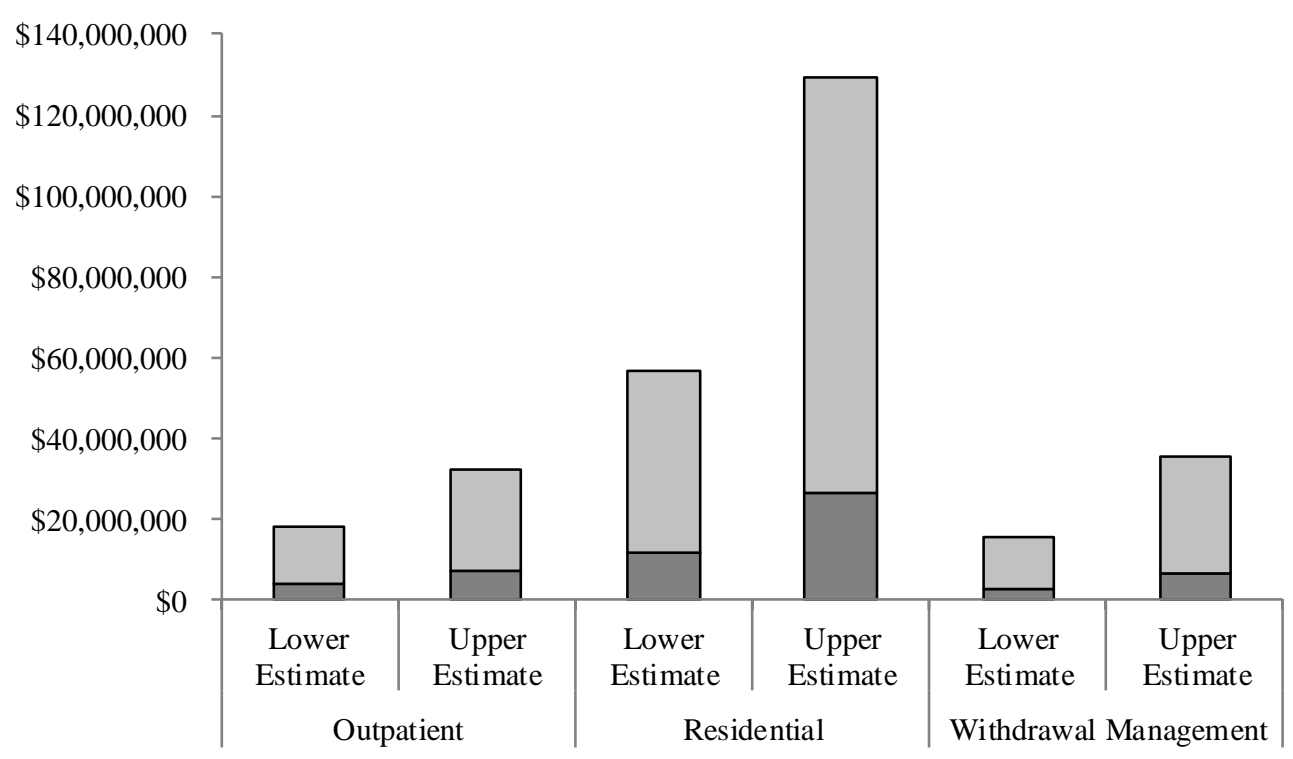

Figure 2

Visits per client in outpatient treatment in 2010/2011 by past year mental disorder, across age groups

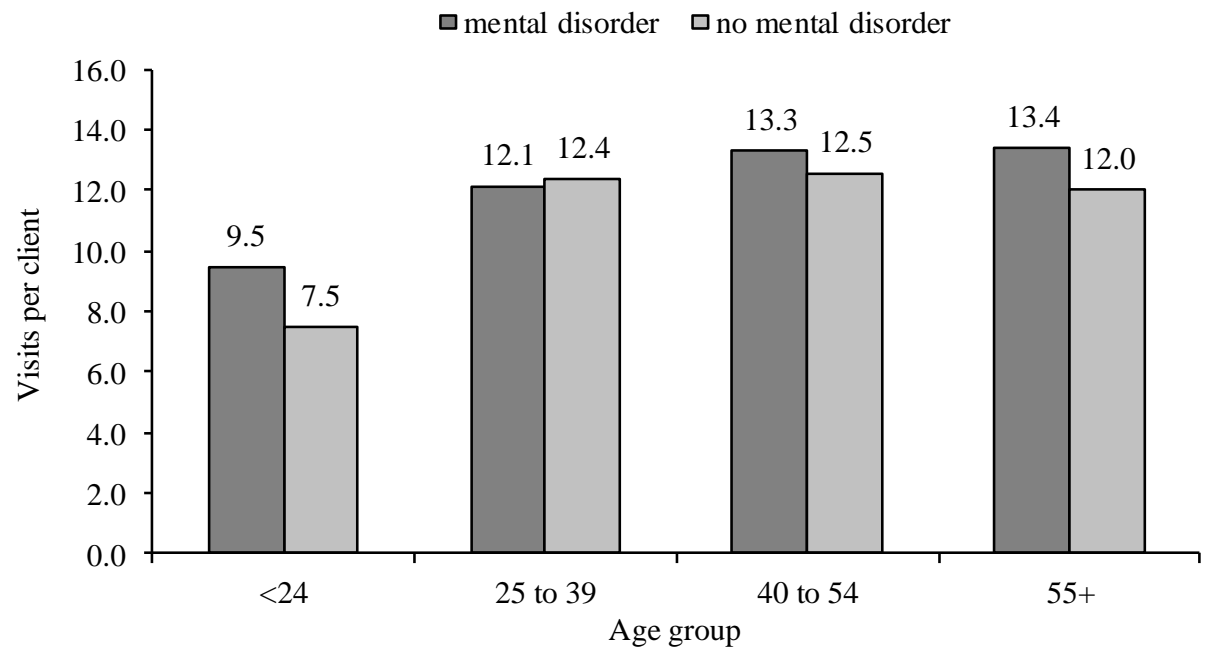




\section{Residential addiction treatment services}

The cost of residential care for clients with mental disorders was estimated to be between \$11.7 and \$26.7 million (Table 1). Clients with mental disorders comprised $23 \%$ of the caseload of residential services, regardless of whether multiple admissions per client were included, and accounted for $21 \%$ of the total costs or volume of residential care delivered during 2010-2011.

Clients with mental disorders spent an average of 48.1 days in residential care in 2010-2011, compared to 54.8 days for those without mental disorders. Among men, the average number of days of care was 52.3 for those with mental disorders and 61.2 for those without. Women spent fewer days in residential care, on average, regardless of their mental disorder status (41 days for both groups). Clients without mental disorders who were between the ages of 25 and 54 had the highest average number of days spent in residential care (Figure 3). The most notable difference between those with and without mental disorders occurred for those aged 40 to 54; clients in this group without mental disorders spent an average of 12 additional days in care.

\section{Residential withdrawal management services}

The cost of residential withdrawal management services in 2010-2011 for clients with mental disorders was estimated to be between $\$ 2.8$ and $\$ 6.4$ million (Table 1). This represents $18 \%$ of the overall volume (in days) of withdrawal management care delivered during the year. By comparison, clients with mental disorders comprised 20\% of the client caseload and $16 \%$ of admissions.
The mean number of days spent in withdrawal management was 6.0 for clients with and 6.8 for clients without mental disorders. These figures were higher for men (6.6 days and 7.2 days for those with and without mental disorders, respectively) than for women (4.8 days and 5.7 days for those with and without mental disorders, respectively). The mean number of days spent in withdrawal management care tended to increase with age (Figure 4). Among those with mental disorders, clients aged 55 and older spent the longest time in treatment, on average. Among those without mental disorders, clients aged 40 to 54 had the highest average number of days in treatment.

\section{Discussion}

This study provides a unique system-level perspective on the implications of co-occurring mental disorders for the course and costs of specialized addiction treatment. In Ontario, approximately one in five clients admitted to addiction treatment programs in 2010-2011 reported pastyear mental disorders. The annual costs of treatment for these clients were estimated to be between $\$ 18.5$ and $\$ 40.3$ million-an amount that was proportionate to their representation in the system. This finding generally held across service categories. Specifically, the proportions of costs attributed to clients with mental disorders varied by only 2 to 4 percentage points from the prevalence of mental disorders within outpatient, residential, and withdrawal management modalities.

\section{Figure 3}

Days of residential treatment per client in 2010-2011 by past-year mental disorder, across age groups

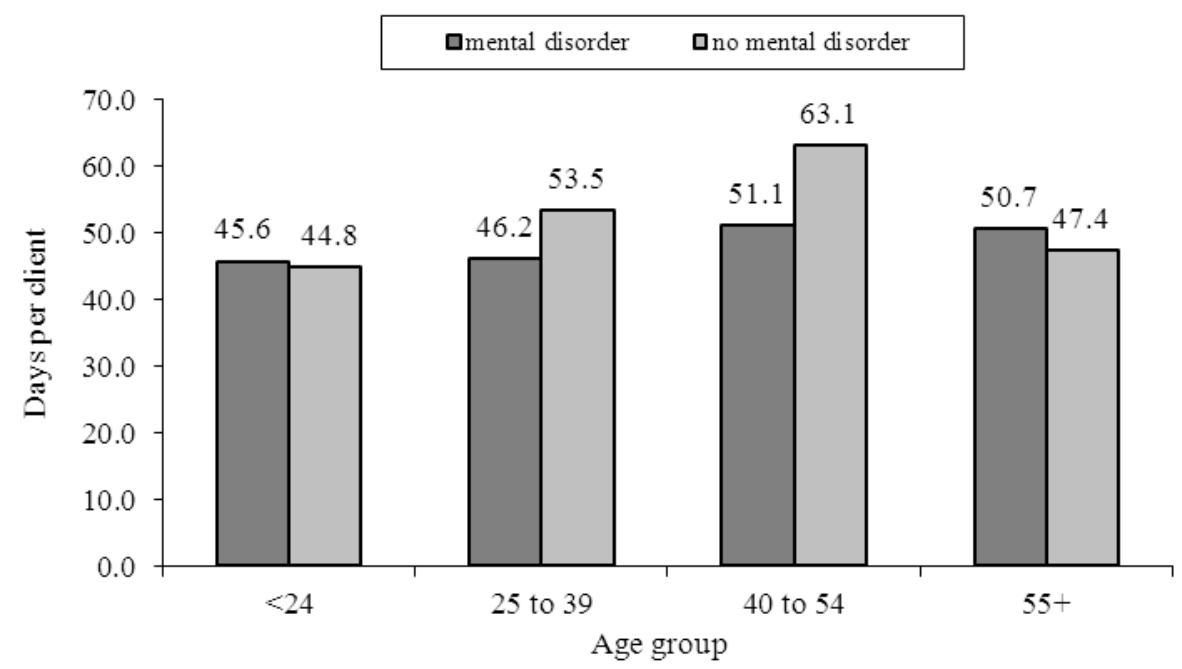


Figure 4

Days of residential withdrawal management treatment per client in 2010/2011 by past year mental disorder, across age groups

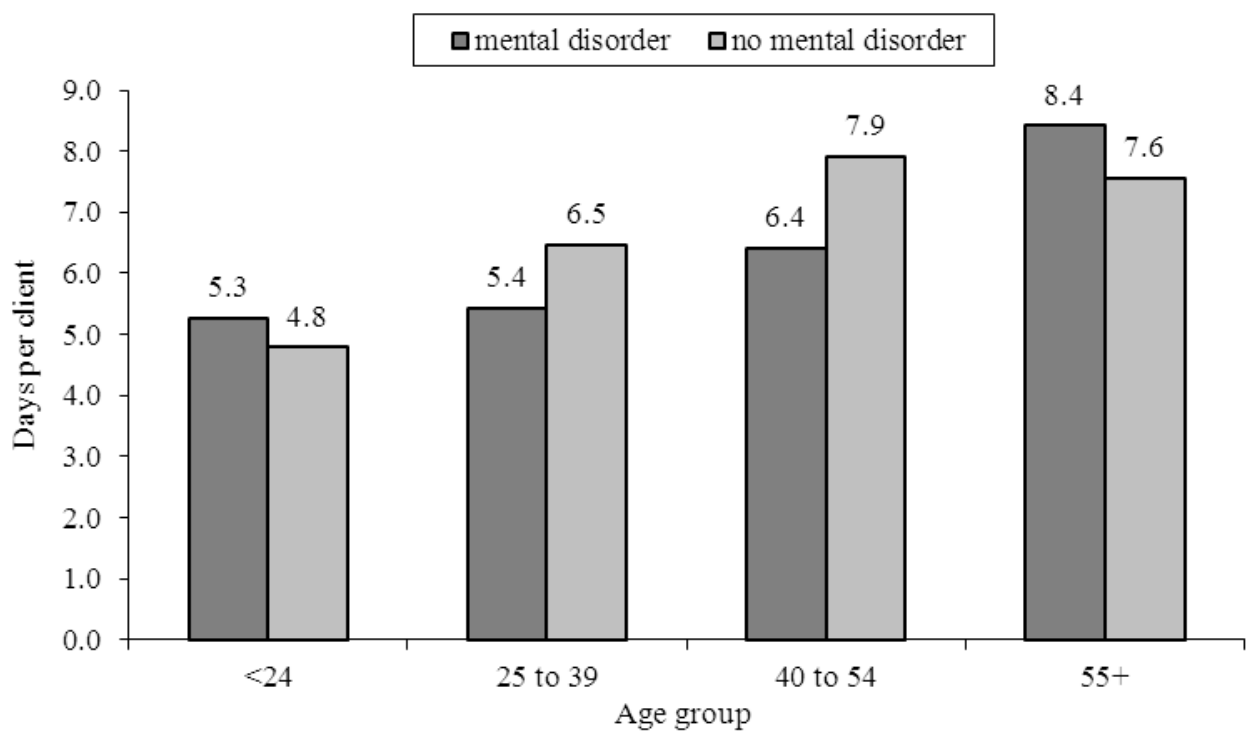

The impact of mental disorders on the volume of care received during 2010-2011 showed somewhat greater differences across service types. On average, a past-year mental disorder was associated with one additional outpatient visit during the year, but one fewer day in withdrawal management. These variations are unlikely to represent clinically meaningful differences in treatment dose. However, a larger difference was seen in the volume of residential care, where having a mental disorder was associated with roughly seven fewer days of care over the year. Even more notable differences between those with and without mental disorders emerged among subgroups defined by sex and age. For instance, the average number of days spent in residential care during the year was lower for women than men, and among women the number of days did not differ for those with and those without mental disorders. As a result, the sex difference in the average number of days spent in residential care was particularly large for clients without a past-year mental disorder: on average, men without mental disorders spent roughly 20 days longer than women in residential care during the year. Given the implications of treatment duration for positive outcomes (Hser, Evans, Huang, \& Anglin, 2004; Hubbard, Craddock, \& Anderson, 2003), the gender difference in time spent in residential care deserves further study.

Across age groups, the average volume of services tended to be lower for clients under 25, regardless of mental disorder status. The finding of lower average service volume among clients with mental disorders in residential care was not seen for clients under 25 or older than 54, but was most apparent for clients aged 40 to 54 . The average number of days per client in withdrawal management care increased with age, which is likely a result of age-related increases in the rates and complexity of comorbid medical issues.

These findings are notable in light of evidence that clients with co-occurring disorders present to treatment with a more complex array of clinical challenges (Baker et al., 2007; Wusthoff et al., 2011). Accordingly, it seems intuitive that these differences would translate into different (and, presumably, more intense) patterns of service use and higher costs of care. The lack of difference in the proportional costs of addiction treatment by past-year mental disorders may reflect the limited resources for mental health assessment and follow-up within Ontario's specialized addiction treatment sector. In order to gain a full understanding of the costs of care for co-occurring disorders, a multisectoral approach (i.e., one that includes mental and general medical services) is needed. Nonetheless, in the context of low system-level integration for specialized mental health and addictions services, including an absence of shared funding and administration, the implications of co-occurring disorders for the volume and costs of services within each care sector remains a relevant question for clinicians, planners, and policy makers.

The use of data representing the entire specialized addiction treatment system in Ontario is a major strength of this analysis. Within Canada, DATIS is unrivalled in its scope and coverage of this sector of care (Beasley, Jesseman, Patton, \& National Treatment Working Group, 2012). However, DATIS does not capture forms of addiction treatment that are provided outside of this specialized system. In addition to methadone maintenance treatment, this would include brief interventions delivered in primary care settings, medications and counselling by mental health 
care providers, private treatment clinics, and self-help or peer support groups (e.g., Alcoholics/Narcotics Anonymous). As well, a full psychiatric screening and assessment would have been preferable to self-reported mental disorder diagnoses, which are subject to recall biases and contingent on prior mental health service use. The prevalence of mental disorders in this treatment population is indeed notably lower than that observed when a structured psychiatric assessment is used (Langås et al., 2012).

Overall, at the system-level in Ontario, clients with mental disorders did not account for a disproportionate amount of the total volume or costs of care delivered in the specialized addiction treatment sector during 2010-2011. Specifically, clients with past-year diagnoses of mental disorders incurred an estimated \$18.5 to \$40.3 million in treatment costs - an amount that was approximately proportional to their representation in the system. Additional analyses revealed more prominent differences in the average volumes of care per client between specific population subgroups; most notably, men without mental disorders had considerably longer stays in residential treatment than women (both with and without mental disorders). While these findings may appear to signal a mismatch between the needs of clients with co-occurring disorders and their use of specialized services, it is also possible that such clients receive additional care in other treatment sectors. Further studies are warranted to explore potential unmet needs among clients with co-occurring disorders in addiction treatment.

\section{Acknowledgements}

The authors would like to thank the DATIS team, especially Brenda Peecock and Daniel Elliot, for their assistance with the data analysis, and Garth Martin, Addiction Consulting Services, for providing the unit costs.

\section{References}

Adamson, S. J., Todd, F. C., Sellman, J. D., Huriwai, T., \& Porter, J. (2006). Coexisting psychiatric disorders in a New Zealand outpatient alcohol and other drug clinical population. Australian and New Zealand Journal of Psychiatry, 40(2), 164-170.

Baker, K. D., Lubman, D. I., Cosgrave, E. M., Killackey, E. J., Yuen, H. P., Hides, L., . . .Yung, A. R. (2007). Impact of co-occurring substance use on 6-month outcomes for young people seeking mental health treatment. Australian and New Zealand Journal of Psychiatry, 41(11), 896-902.

Beasley, E., Jesseman, R., Patton, D., \& National Treatment Indicators Working Group. (2012). National Treatment Indicators report, 2012. Ottawa, ON: Canadian Centre on Substance Abuse.

Bradizza, C. M., Stasiewicz, P. R., \& Paas, N. D. (2006). Relapse to alcohol and drug use among individuals diagnosed with co-occurring mental health and substance use disorders: A review. Clinical Psychology Review, 26(2), 162-178.

Carroll, K. M., Power, M. D., Bryant, K., \& Rounsaville, B. J. (1993). One-year follow-up status of treatmentseeking cocaine abusers. Journal of Nervous and Mental Disease, 181(2), 71-79.

Castel, S., Rush, B. R., Urbanoski, K., \& Toneatto, T. (2006). Overlap of clusters of psychiatric symptoms among clients of a comprehensive addiction treatment service. Psychology of Addictive Behaviors, 20(1), 2835.

Chan, Y.-F., Dennis, M. L., \& Funk, R. R. (2008). Prevalence and comorbidity of major internalizing and externalizing problems among adolescents and adults presenting to substance abuse treatment. Journal of Substance Abuse Treatment, 34, 14-24.

Chi, F. W., Satre, D. D., \& Weiser, C. (2006). Chemical dependency patients with co-occurring psychiatric diagnoses: Service patterns and 1-year outcomes. Alcoholism: Clinical and Experimental Research, 30(5), 851-859.

Compton, W. M., Cottler, J. B., Jacobs, J. L., BenAbdallah, A., \& Spitznagel, E. L. (2003). The role of psychiatric disorders in predicting drug dependence treatment outcomes. American Journal of Psychiatry, 160(5), 890-895.

Grant, B. F., Stinson, F. S., Dawson, D. A., Chou, S. P., Dufour, M. C., Compton, W., .. . Kaplan, K. (2004). Prevalence and co-occurrence of substance use disorders and independent mood and anxiety disorders. Archives of General Psychiatry, 61(8), 807816.

Hoff, R. A., \& Rosenheck, R. A. (1999). The cost of treating substance abuse patients with and without comorbid psychiatric disorders. Psychiatric Services, 50(10), 1309-1315.

Hser, Y. I., Evans, E., Huang, D., \& Anglin, D. M. (2004). Relationship between drug treatment services, retention, and outcomes. Psychiatric Services, 55(7), 767-774.

Hubbard, R. L., Craddock, S. G., \& Anderson, J. (2003). Overview of 5-year follow-up outcomes in the drug abuse treatment outcome studies (DATOS). Journal of Substance Abuse Treatment, 25(3), 125-134.

Kessler, R. C., Crum, R. M., Warner, L. A., Nelson, C. B., Schulenberg, J., \& Anthony, J. C. (1997). Lifetime cooccurrence of DSM-III-R alcohol abuse and dependence with other psychiatric disorders in the National Comorbidity Survey. Archives of General Psychiatry, 54(4), 313-321.

Langås, A.-M., Malt, U. F., \& Opjordsmoen, S. (2012). Substance use disorders and comorbid mental disorders in first-time admitted patients from a catchment area. European Addiction Research, 18(1), 16-25.

Ogborne, A. C., Braun, K., \& Rush, B. R. (1998). Developing an integrated information system for specialized addiction treatment agencies. Journal of Behavioral Health Services \& Research, 25(1), 100107.

Pani, P. P., Maremmani, I., Pacini, M., Lamanna, F., Maremmani, A. G., \& Dell'osso, L. (2011). Effect of 
psychiatric severity on the outcome of methadone maintenance treatment. European Addiction Research, 17(2), 80-89.

Popova, S., Rehm, J., \& Fischer, B. (2006). An overview of illegal opioid use and health services utilization in Canada. Public Health, 120(4), 320-328.

Popova, S., Rehm, J., Patra, J., Baliunas, D., \& Taylor, B. (2007). Illegal drug-attributable morbidity in Canada 2002. Drug and Alcohol Review, 26(3), 251-263.

Rehm, J., Baliunas, D., Brochu, S., Fischer, B., Gnam, W., Patra, J., . . Taylor, B. (2006). The costs of substance abuse in Canada 2002. ISBN 1-897321-10-4. Ottawa, ON: Canadian Centre on Substance Abuse.

Rehm, J., Gnam, W., Popova, S., Baliunas, D. Brochu, S., Fischer, B., . . . Taylor, B. (2007). The social costs of alcohol, illegal drugs and tobacco in Canada 2002. Journal of Studies on Alcohol and Drugs, 68(6), 886895.

Rotondi, N. K., \& Rush, B. (2012). Monitoring utilization of a large scale addiction treatment system: The Drug and Alcohol Treatment Information System (DATIS). Substance Abuse: Research and Treatment, 6, 73-84.

Rush, B. R., Urbanoski, K. I., Bassani, D., Castel, S., Wild, T. C., Strike, C., . . . Somers, J. (2008). Prevalence of co-occurring substance use and other mental disorders in the Canadian population. Canadian Journal of Psychiatry, 53(12), 800-809.

Stark, M. (1992). Dropping out of substance abuse treatment: A clinically oriented review. Clinical Psychology Review, 12(1), 93-116.

Teesson, M., Hall, W., Lynskey, M., \& Degenhardt, L. (2000). Alcohol and drug-use disorders in Australia: Implications of the National Survey of Mental Health and Wellbeing. Australian and New Zealand Journal of Psychiatry, 34(2), 206-213.

Verthein, U., Degkwitz, P., Haasen, C., \& Krausz, M. (2005). Significance of comorbidity for the long-term course of opiate dependence. European Addiction Research, 11(1), 15-21.

Weisner, C., Matzger, H., \& Kaskutas, L. A. (2003). How important is treatment? One-year outcomes of treated and untreated alcohol-dependent individuals. Addiction, 98(7), 901-911.

Wüsthoff, L. E., Waal, H., Ruud, T., \& Gråwe, R. W. (2011). A cross-sectional study of patients with and without substance use disorders in community mental health centres. BMC Psychiatry, 11, 93. 